\title{
Hyaluronan preserves the proliferation and differentiation potentials of long-term cultured murine adipose-derived stromal cells
}

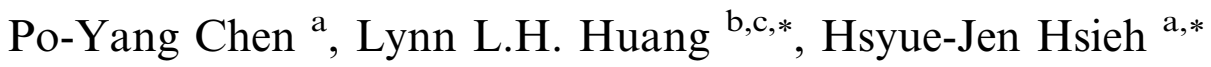 \\ a Department of Chemical Engineering, National Taiwan University, No.1, Sec. 4, Roosevelt Road, Taipei 10617, Taiwan \\ b Institute of Biotechnology, National Cheng Kung University, 1 University Road, Tainan 70101, Taiwan \\ ${ }^{\mathrm{c}}$ Institute of Clinical Medicine, National Cheng Kung University, Tainan 70101, Taiwan
}

Received 7 April 2007

Available online 8 June 2007

\begin{abstract}
For long-term culture, murine adipose-derived stromal cells (mADSCs) at latter passages demonstrated a marked decline in proliferative activity, exhibited senescent morphology and reduced differentiation potentials, particularly osteogenesis. To extend the lifespan of mADSCs, two culture conditions containing hyaluronan (HA) was compared in our study, one as a culture medium supplement (SHA), and the other where HA was pre-coated on culture surface (CHA). mADSCs cultivated with SHA exhibited a prolonged lifespan, reduced cellular senescence, and enhanced osteogenic potential compared to regular culture condition (control). Upon CHA treatment, mADSCs tended to form cell aggregates with gradual growth profiles, while their differentiation activities remained similar to SHA groups. After transferring mADSCs from CHA to control surface, they were shown to have an extended lifespan and an increase of osteogenic potential. Our results suggested that HA can be useful for preserving the proliferation and differentiation potentials of long-term cultured mADSCs.
\end{abstract}

(c) 2007 Elsevier Inc. All rights reserved.

Keywords: Hyaluronan; Adipose-derived stromal cell; Proliferation; Differentiation

The murine adipose-derived stromal cells (mADSCs) have differentiation capacity toward a variety of lineages [1-3]. For in vitro cultures, mADSCs exhibit a finite proliferative capacity and acquire senescent morphology rapidly in our preliminary study. It is possible that the murine cells are highly sensitive to environmental stresses, such as those induced by frequent subcultivation $[4,5]$ or the hyperoxic condition in vitro [6]. This may result in impaired differentiation capability similar to subcultured mesenchymal stem cells reported [7].

For extending the lifespan of mADSCs, hyaluronan (HA) may be applied since it has been reported to influence

\footnotetext{
* Corresponding authors. Fax: +886 62080165 (L.L. Huang), +886 6 23623040 (H.-J. Hsieh).

E-mail addresses: lynn@mail.ncku.edu.tw (L.L.H. Huang), hjhsieh@ ntu.edu.tw (H.-J. Hsieh).
}

the adhesion, migration, proliferation [8,9], and the cell fate determination of mesenchymal cells [10] as well as the developmental capacity of embryos in vitro [11]. The enhancement of osteogenic potential of rat osteoblasts by an initial administration of HA during first plating was suggested [12]. Other findings also suggested that HA can stimulate the proliferation of primary porcine bone marrow stromal cells during early passage [13]. Given the various functions of HA, little was known about the effects of long-term exposure of HA on the propagation and differentiation of adult stem cells. Thus, we aimed to assess the effects of HA on the feasibility of in vitro expansion and the preservation of differentiation capacity of long-term cultured mADSCs by comparing the culture of mADSCs on a HA pre-coated microenvironment with that of HA presented in medium where the regular culture surface serves as a control. 


\section{Materials and methods}

Isolation and culture of $M A D S C s$. mADSCs were isolated as previously described [2]. Male FVB/N mice were housed and cared under standard conditions according to institutional guidelines for animal regulation at the National Cheng Kung University. Briefly, the inguinal fat pads from FVB/N mice were harvested and washed with phosphate buffered saline (GibcoBRL, Grand Island, USA). They were then finely minced and digested with $0.1 \%$ collagenase (Worthington, Lakewood, USA) at $37^{\circ} \mathrm{C}$ for $45 \mathrm{~min}$. An equal volume of Dulbecco's modified Eagle's medium (DMEM, GibcoBRL) containing 10\% fetal bovine serum (FBS, Biological Industries, Israel) was added and the resulting solution was filtered through a $100-\mu \mathrm{m}$ mesh, followed by centrifugation at $250 \mathrm{~g}$ for $10 \mathrm{~min}$. The pellet was collected and resuspended in $160 \mathrm{mM} \mathrm{NH}_{4} \mathrm{Cl}$ (Sigma, USA) to lyse the red blood cells and spun at $250 \mathrm{~g}$ for $10 \mathrm{~min}$. The cell pellet was collected and resuspended in conventional culture medium of DMEM-10\% FBS containing 1\% antibiotic/antimycotic solution or in addition of indicated HA-containing medium. The cell suspensions were then plated at $1 \times 10^{4}$ cells $/ \mathrm{cm}^{2}$ on regular culture surface or on HA precoated surface and incubated at $37^{\circ} \mathrm{C}$ with $5 \% \mathrm{CO}_{2}$.

mADSCs cultured in regular and HA-containing culture conditions. mADSCs cultured with DMEM-10\% FBS on regular culture surface were used as the control. Two kinds of HA-containing culture system were applied: (A) SHA where mADSCs were cultivated with DMEM-10\% FBS containing HA ( $M_{\mathrm{w}}=720 \mathrm{kDa}$, Pentapharm, Basel, Switzerland $)$ at concentration of $0.2 \mathrm{mg} / \mathrm{mL}$ (SHA0.2) or $0.05 \mathrm{mg} / \mathrm{mL}$ (SHA0.05) on regular culture surface; (B) CHA where mADSCs were cultivated with DMEM$10 \%$ FBS on HA pre-coated surfaces containing $5 \mu \mathrm{g} / \mathrm{cm}^{2}$ (CHA5) or $20 \mu \mathrm{g} / \mathrm{cm}^{2}$ (CHA20) of HA. Serial passages of mADSCs cultured in control, SHA, and CHA were carried out when cells reach confluence. mADSCs were trypsinized, centrifuged and resuspended in appropriate culture medium, i.e., DMEM-10\% FBS for control and CHA groups; DMEM-10\% FBS-SHA for SHA groups. The cells were then plated at $1 \times 10^{4}$ cells $/ \mathrm{cm}^{2}$ in each group. The increase of population doubling $(\Delta \mathrm{PD})$ was calculated according to the formula of $\Delta \mathrm{PD}=\log \left(N_{\mathrm{f}} / N_{0}\right) / \log 2$, where $N_{\mathrm{f}}$ is the final number of cells at subconfluence, and $N_{0}$ is the initial number of plated cells.

Transfer culture. mADSCs initially cultured on CHA20 for 3 and 5 passages were subcultured into regular culture surface. The term "CHA_P3/C" and "CHA_P5/C" denotes the transfer and subculture of mADSCs from CHA20 at $\mathrm{P} 3$ and at $\mathrm{P} 5$, respectively, to regular culture surface. "P3+X" denotes that mADSCs were cultured on CHA20 for three passages and then cultured on regular culture surface for " $\mathrm{X}$ " passages. $\triangle \mathrm{PD}$ was calculated as above.

5-Bromo-2-deoxyuridine (BrdU) incorporation assay. BrdU incorporation assay was performed in control, SHA0.05, SHA0.2, CHA5, and CHA20 groups for passage numbers of 1,3 , and 5. After plating for $12 \mathrm{~h}$, mADSCs were incubated with medium containing $10 \mu \mathrm{M}$ BrdU (Sigma) for $24 \mathrm{~h}$. The mADSCs were then washed twice with phosphate buffered saline (PBS; Sigma) and once with $70 \%$ ethanol, hydrolyzed in $1 \mathrm{M}$ icecold $\mathrm{HCl}$ for $10 \mathrm{~min}$, followed by $2 \mathrm{M} \mathrm{HCl}$ at room temperature for another $10 \mathrm{~min}$, and incubated with anti-BrdU antibody (BD Pharmingen) for $1 \mathrm{~h}$ at room temperature. After incubation with horseradish peroxidase-conjugated $\mathrm{IgG}_{1}$ (BETHÝL Laboratories, USA) at room temperature for $30 \mathrm{~min}$, 3-amino-9-ethylcarbazole (Zymed, USA) was used as a substrate. Hematoxylin was used to counterstain the non-mitotic cells. The percentage of BrdU-incorporated cells was assessed by counting BrdUpositive nuclei versus total nuclei in 10 different microscopic fields for each triplicate sample.

Senescence-associated $\beta$-galactosidase ( $S A$ - $\beta$-gal) activity. The detection of SA- $\beta$-gal activity was performed according to previous published method [14]. Briefly, mADSCs cultured in each condition for 1, 3, and 5 passages were fixed by $4 \%$ paraformaldehyde and then incubated with staining solution of $1 \mathrm{mg} / \mathrm{mL} 5$-bromo-4-chloroindol-3-yl $\beta$-D-galactopyranoside (X-Gal; Sigma) in $40 \mathrm{mM}$ citric acid-sodium phosphate buffer ( $\mathrm{pH}$ 6.0) containing $5 \mathrm{mM}$ potassium ferrocyanide, $5 \mathrm{mM}$ potassium ferricyanide, $150 \mathrm{mM} \mathrm{NaCl}$, and $2 \mathrm{mM} \mathrm{MgCl}_{2}$ for $16 \mathrm{~h}$ until a visible green color was present around the nuclei. The cells were then counterstained with hematoxylin. The percentage of senescent cells was assessed by counting SA- $\beta$-gal positive nuclei versus total nuclei in 10 different microscopic fields for each triplicate sample.

Differentiation. Adipogenic and osteogenic inductions of mADSCs were carried out according to the procedures reported by Zuk et al. (2001) with minor modifications [15]. For differentiation, mADSCs at each passage, and under SHA and CHA treatment were initially plated at $10^{4} / \mathrm{cm}^{2}$ and cultured for 3 days prior to induction. For adipogenic induction, mADSCs were cultured in DMEM-10\% FBS supplemented with indomethacin, dexamethasone, methyl-isobutylxanthine, and insulin for 7 days, followed by maturation medium of DMEM- $10 \%$ FBS and $10 \mu \mathrm{g} /$ $\mathrm{mL}$ insulin for another 7 days. Adipogenesis was assessed by oil red $\mathrm{O}$ staining. For osteogenic differentiation, mADSCs were cultured in DMEM-10\% FBS, $\beta$-glycerophosphate, dexamethasone, and ascorbic acid-2-phosphate for at least 2 weeks. Alkaline phosphatase activity and matrix calcification were examined at day 7 and 14, respectively. Alkaline phosphatase activity was detected by incubating the live cells with staining solution ( $1 \%$ naphthol AS-BI phosphate and $1 \mathrm{mg} / \mathrm{mL}$ fast red TR salts) at $37^{\circ} \mathrm{C}$ for $20 \mathrm{~min}$. For matrix calcification, cells were fixed prior to vonKossa staining. For quantifying the degree of osteogenesis, the cells were stained with silver nitrate only, and the calcium deposition regions from 10 microscopic fields were assessed and calculated by Sigma Scan Pro (SPSS Inc.) for each triplicate sample.

$R T-P C R$ analysis. Total RNA from the groups of control, SHA0.2, CHA20, and CHA20/C at various passages of P1, P3, P5, and P7 was extracted with TRI ${ }^{\circledR}$ reagent (Sigma), and the cDNA was synthesized with reverse transcriptase (RT) system (Promega, Madison, USA) according to the manufacturer's protocol. Primer pairs of p16 $6^{\mathrm{INK} 4 \mathrm{a}}: 5^{\prime}$-GCAGCATGG AGTCCGCTG CAGACA-3' (sense) and 5'-CGGATTTAGCTCTGCT CTTGGGAT-3' (antisense) [16], and glyceraldehyde-3-phosphate dehydrogenase (G3PDH): 5'-ACCACAGTCCATG CCATCAC-3' (sense) and 5'-TCCACCACCCTGTTGCT GTA-3' (antisense) [17] were synthesized. The amount of each template was adjusted to give PCR signal at the exponential phase. Amplified PCR products were electrophoresed on a $2 \%$ agarose gel and visualized with ethidium bromide.

Statistical analysis. Student's $t$ test was used to calculate the $p$ values.

\section{Results}

\section{Morphological change and impaired differentiation potential through subcultivation}

In primary cultures, mADSCs seeded on regular culture surface (we termed "control" hereafter) exhibited a spindle, fibroblastic morphology and initially proliferated at a high growth rate. As subcultivation proceeded, a progressive decrease in growth rate of mADSCs was soon observed at around the 4th passage ( $\mathrm{P} 4)$ in our preliminary study. In this state, the cells became larger and flatter in morphology resembling the reported senescent fibroblasts and were stained positive for the activity of SA- $\beta$-gal at $\mathrm{pH} 6.0$, indicating that the cells had undergone cellular senescence (Fig. 1A). We further investigated the multilineage potentials of mADSCs in these different passages. The mADSCs from the first passage (P1) and the fifth passage (P5) were incubated with osteogenic or adipogenic induction medium for 14 days (Fig. 1A). Upon osteogenic induction, the mADSCs from P1 showed much more matrix calcification (vonKossa stain) than those from P5. In the case of adipogenic induction, most of the cells from P1 were stained positive for oil red $\mathrm{O}$, while only few cells from $\mathrm{P} 5$ were stained positive. 
A Light
microscopy

SA- $\beta$-gal/

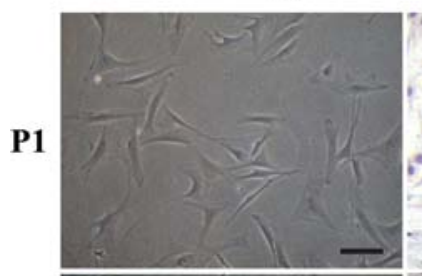

Hematoxylin

Osteogenic/

Von Kossa

Adipogenic/
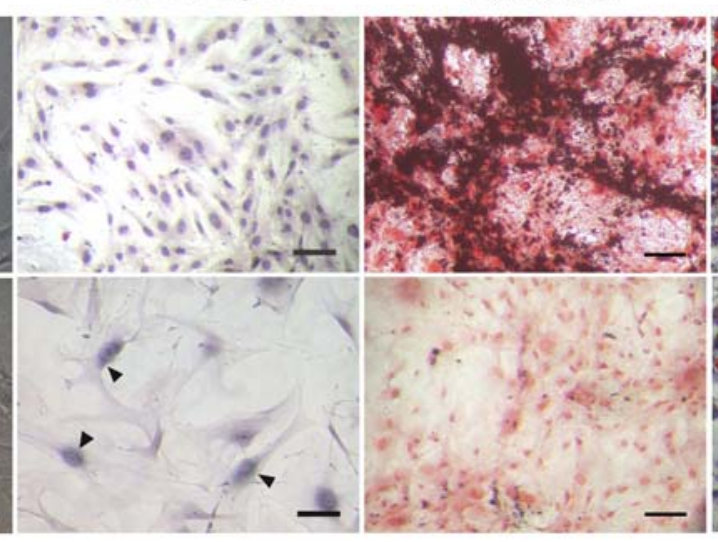

Oil Red 0

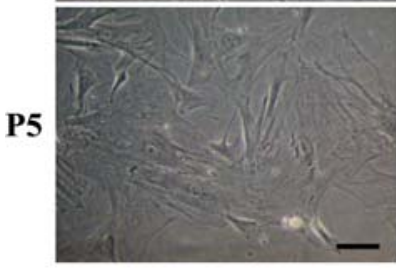

B

SHA
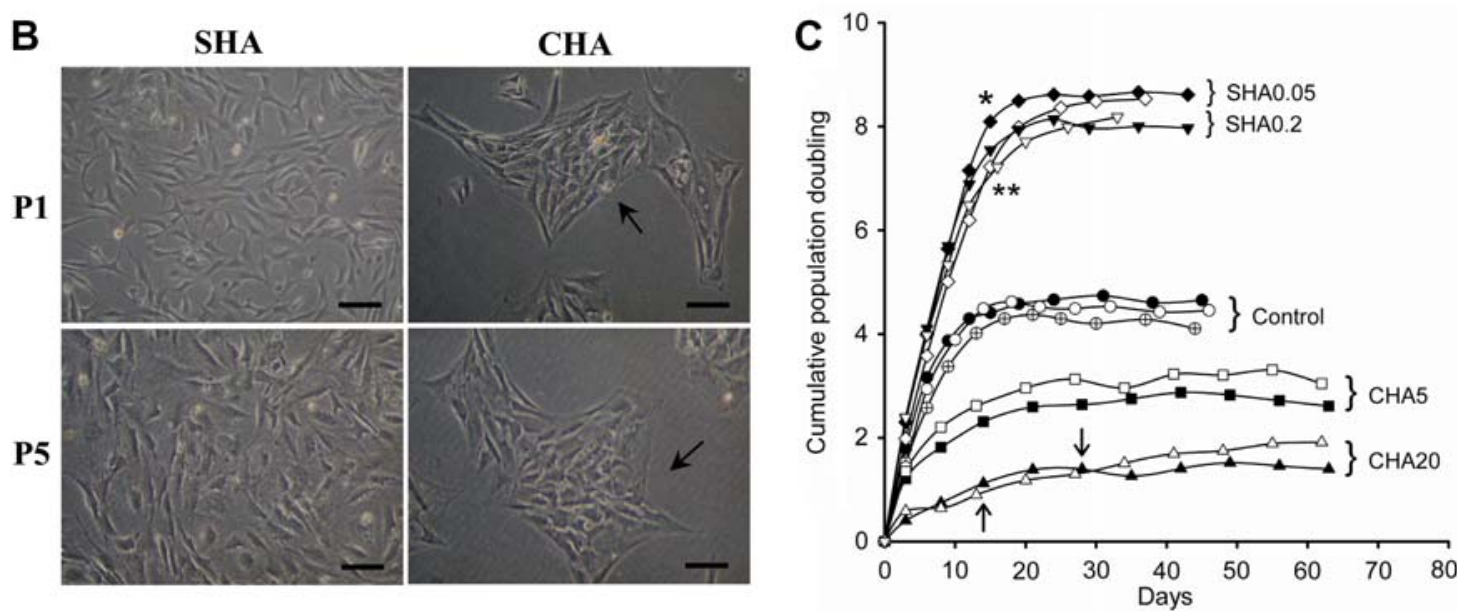

Fig. 1. (A) Morphology and differentiation potentials of mADSCs on regular culture surface (control). mADSCs were harvested and subcultured as described in Materials and methods. Cell morphology, staining of SA- $\beta$-gal activity as well as osteogenic and adipogenic differentiation potentials of mADSCs were examined at the first passage (P1) and the fifth passage (P5), respectively. Osteogenic and adipogenic induction at P1 and P5 were carried out for 14 days, and von Kossa staining was applied to assess the degree of matrix calcification while oil red O staining was used to examine the adipogenic potentials. Bar $=100 \mu \mathrm{m}$. (B) Morphology of mADSCs upon HA treatment. mADSCs were harvested and cultured under two different HA-containing conditions (SHA and CHA, respectively) for one and five passages (P1 and P5, respectively). Arrows, mADSCs formed aggregate on CHA surface. $\mathrm{Bar}=100 \mu \mathrm{m}$. (C) Proliferative lifespan of mADSCs cultured on control surface, with SHA $(0.05$ and $0.2 \mathrm{mg} / \mathrm{mL}) \mathrm{and} \mathrm{CHA}\left(5 \mathrm{and} 20 \mu \mathrm{g} / \mathrm{cm}^{2}\right)$, respectively. The drawing represents the cumulative population doublings with time where each dot represents one passage. Three independent experiments were performed in control group, while two independent experiments were performed in each of SHA0.05, SHA0.2, CHA5, and CHA20 groups. Arrows, the passage number (P3 and P5) where a transfer culture from CHA20 to regular culture surface was carried out. ${ }^{*} p<0.05$ (SHA0.05) and ${ }^{* *} p<0.01$ (SHA0.2) versus control at P5.

\section{Altered proliferative behaviors of $m A D S C$ s in response to $H A$}

Administration of HA in different ways (SHA and CHA) for culturing mADSCs exhibited altered proliferative behaviors. The mADSCs cultured on CHA tended to form cell aggregates even through latter passages (P5), whereas mADSCs cultured with SHA spread well on the culture surface (Fig. 1B). Interestingly, fewer cells at P5 were found morphologically senescent in both SHA and CHA groups in comparison to the control group (Fig. 1A). The proliferative lifespans of mADSCs in each condition were compared in Fig. 1C. The growth rate of mADSCs cultured with SHA0.05 and SHA0.2 were significantly higher $(p<0.05$ and $p<0.01$, respectively, at P5) than that of control group. However, the differences between SHA0.05 and SHA0.2 group were insignificant. Upon culturing on CHA, mADSCs exhibited a much more gradual growth profile and almost no increase in cell numbers at each passage after P5. In addition, the proliferative lifespan seemed to be shorter with increasing amount of CHA (CHA20 versus CHA5).

BrdU incorporation for $24 \mathrm{~h}$ was carried out at P1, P3, and $\mathrm{P} 5$, and the percentages of BrdU-incorporating cells were calculated (Fig. 2A). In each group, more cells incorporated BrdU were found at early passage (P1) than at latter passage (P5). At each passage, even at P5, the percentage of BrdU-incorporating cells were significantly higher in SHA groups $(p<0.05)$. Surprisingly, the amounts of BrdU-incorporating mADSCs in CHA groups at each 
A

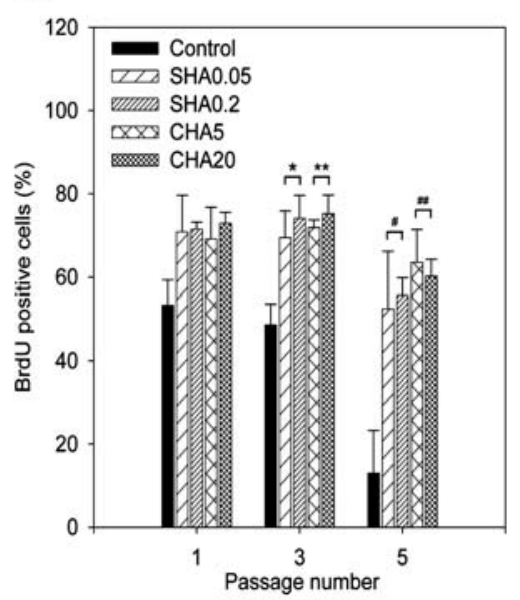

B

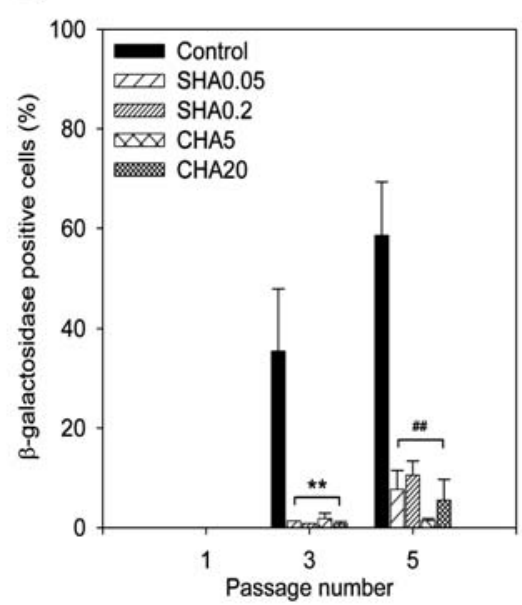

C

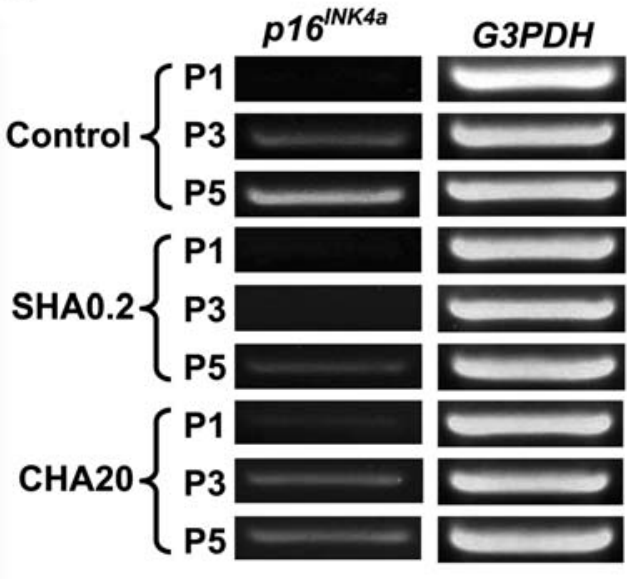

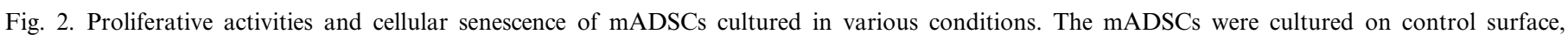

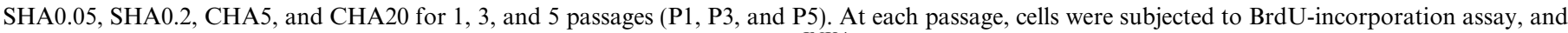

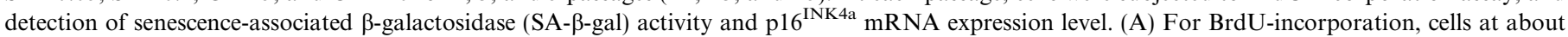

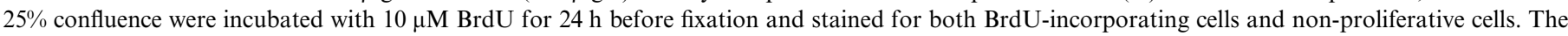

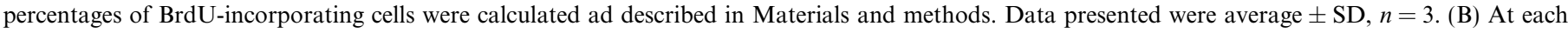

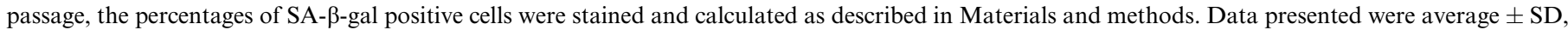

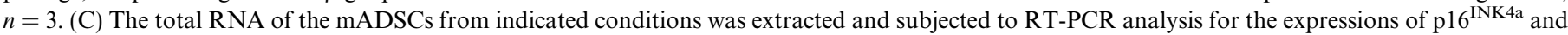
G3PDH. In (A,B), ${ }^{*} p<0.05$ and ${ }^{* *} p<0.01$ versus control group of P3, and ${ }^{\#} p<0.05$ and ${ }^{\# \#} p<0.01$ versus control group of P5.

passage were shown to be significantly higher than that of the control group $(p<0.01)$. The differences between SHA and CHA groups were insignificant.

\section{Reduced senescence of $M A D S C$ s by $H A$}

In addition to the assay of proliferative activity, the senescence of mADSCs in each group was further investigated by staining for the SA- $\beta$-gal activity along with the progression of serial passages, and the percentages of senescent cells were counted as shown in Fig. 2B. In the control group, indeed, the percentage of senescent cells increased with increasing passage numbers and the phenomenon was most profound, i.e., $0 \%$ at P1 and $58.5 \pm 10.7 \%$ at P5. On the contrary, significantly fewer mADSCs were found senescent in the SHA and CHA groups at $\mathrm{P} 5(p<0.01)$, suggesting that a suppression of senescence was done by administration of HA either through coating on the plate or as a medium supplement.

Several anti-proliferative responses including the buildup of senescent program have been showed to be mediated by $\mathrm{p} 16^{\mathrm{INK} 4 \mathrm{a}}[18,19]$, a cyclin-dependent kinase inhibitor (CDKI), which induces cell arrest in G1 phase of the cell cycle. We then carried out RT-PCR analysis to examine p16 ${ }^{\mathrm{INK} 4 \mathrm{a}}$ mRNA levels in mADSCs (Fig. 2C). The expression of $\mathrm{p} 16^{\mathrm{INK} 4 \mathrm{a}}$ gene was absent at $\mathrm{P} 1$, and increased along with passage number in the control group. mADSCs of SHA0.2 group were shown to express significantly lower level of $\mathrm{p} 16^{\mathrm{INK} 4 \mathrm{a}}$ in comparison to the control group. Slight increases in $\mathrm{p} 16^{\mathrm{INK} 4 \mathrm{a}}$ transcript were observed in the CHA20 group in comparison to the SHA0.2 group.

\section{Prolongation of lifespan after preculturing on $\mathrm{CHA}$}

Above results revealed that mADSCs cultured on CHA had a slower progression in population doublings with a higher BrdU-incorporating capability. Thus, to further elucidate the effects of CHA, mADSCs initially cultured on CHA20 for three and five passages (indicated by arrows in Fig. 1C) were then transferred to regular culture surface and subcultured for passages. The morphology of mADSCs after transferring remained fibroblastic for at least 5 passages $(\mathrm{P} 3+5)$, with some cells being larger and flatter at $\mathrm{P} 3+7$ (data not shown). A higher $\Delta \mathrm{PD}$ was observed in CHA_P3/C groups, while the cells failed to proliferate in CHA_P5/C groups (Fig. 3). The lifespan of mADSCs in CHA_P3/C groups was shown to extend to 6-10 passages (Fig. 3, up and down arrows).

\section{Preservation of osteogenic potential after pre-conditioning with $H A$}

As subcultivation was performed or senescence developed, mADSCs lost their differentiation capability progressively (Fig. 1A). From above, a retardation of senescent program seemed to be accomplished by HA treatment, the mADSCs undergone various HA treatments for 1 and 5 passages were subjected to osteogenic induction. During differentiation at P5, mADSCs of both SHA and CHA groups deposited sufficient amounts of calcium visible in black after silver nitrate staining on day 7 postinduction (Fig. 4A). The silver nitrate stain-positive regions were quantified on day 14 as shown in Fig. 4B, where the degree of osteogenic differentiation of mADSCs was found 


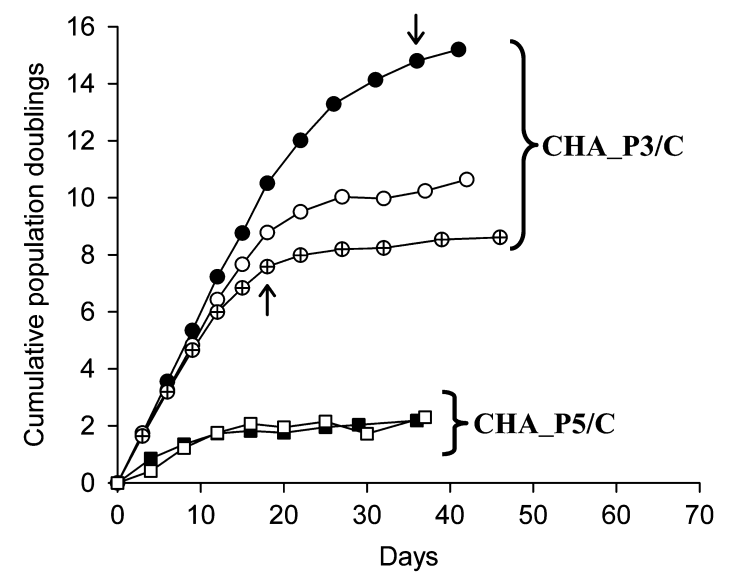

Fig. 3. Proliferative lifespan of mADSCs in response to the transfer culture from CHA20 to control surface. The mADSCs were initially cultured on CHA20 for 3 and 5 passages, and then transferred to control surface for subsequent cultures. The cumulative population doublings of mADSCs after transferring to control surface were calculated and plotted versus time with each dot representative of one passage. Three independent experiments were carried out in CHA_P3/C while two were carried out in CHA_P5/C. In one experiment, the lifespan of the mADSCs extended to 10 passages, i.e., 36 days (arrow down), while the other one extended to only about 6 passages, i.e., 18 days (arrow up).

to be preserved or even significantly enhanced upon both SHA and CHA treatments at either P1 or P5 $(p<0.01$ at least). In addition, the osteogenic potential of transferred mADSCs which was pre-incubated with CHA20 (CHA_P3/C) was also enhanced even at P3+7 (Fig. 4A and $\mathrm{B})$. From the results, mADSCs pre-conditioned by SHA, CHA as well as by transfer culture of CHA_P3/C demonstrated a preservation of osteogenic potential at latter passages (P5) and the differences between HA pre-conditioned groups and control group were shown to be significant $(p<0.01$ at least).

\section{Discussion}

HA is crucial in regulating a variety of cellular responses and it is considered to be a predominant ECM component in bone marrow which forms the niche for hematopoietic stem cells [20], suggesting that HA might be effective in promoting proliferation of stem cells and maintaining them in an undifferentiated state. The current study showed that mADSCs underwent senescence rapidly in regular culture conditions. However, the supply of HA into the medium (SHA) can increase the growth rates of mADSCs at early passages and contributed to the extension of their lifespan with a marked reduction of cellular senescence during subcultivation, indicating that HA seems to be effective in maintaining mADSCs at a proliferative state and delaying the development of senescent program.

We also investigated the effects of CHA on mADSCs and observed the formation of cell aggregates with a much more gradual growth profile during entire culture period (Fig. 2). At latter passages, such aggregation limited the
A
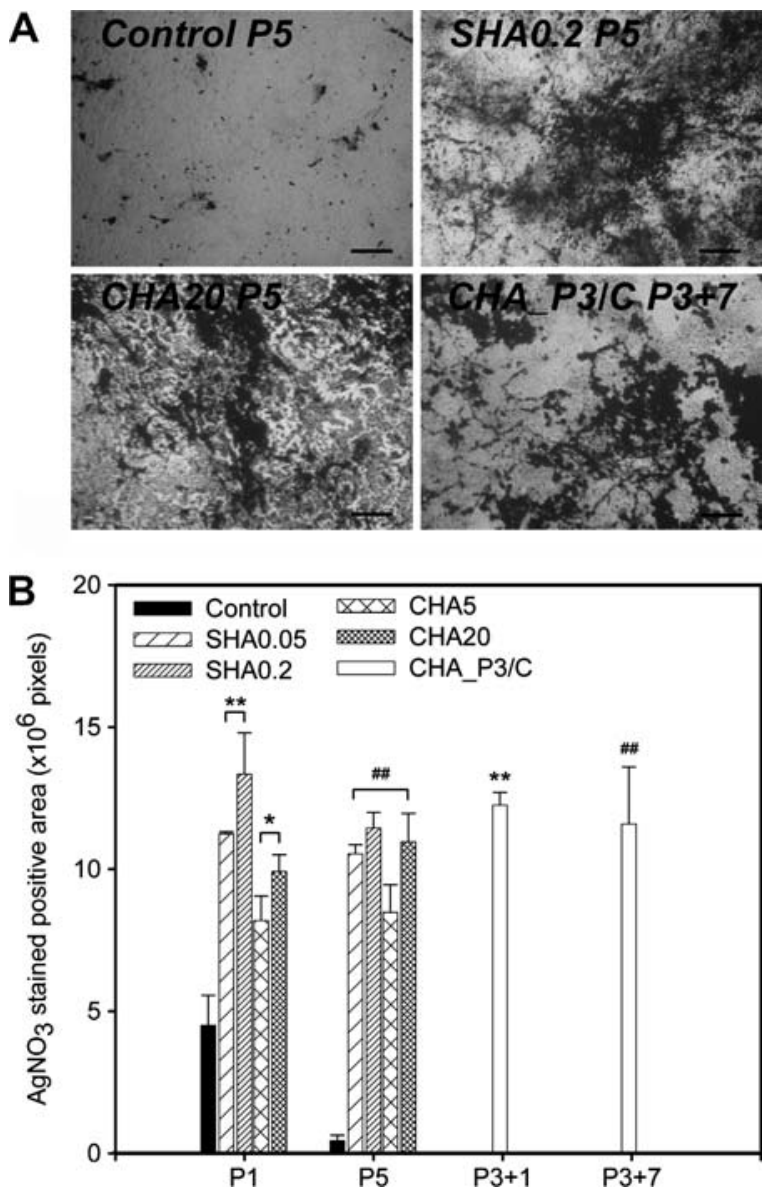

Fig. 4. Osteogenic potentials of mADSCs from control, SHA0.05, SHA0.2, CHA5, CHA20 as well as CHA_P3/C. (A) The mADSCs cultured on control, SHA0.2, and CHA20 for 5 passages and the mADSCs derived from CHA_P3/C at 7th passages $(\mathrm{P} 3+7)$ were incubated with osteogenic induction medium for 14 days. The cells were then fixed and the extent of matrix calcification was examined by silver nitrate staining where the calcium deposition region is shown in black. (B) The extent of osteogenesis was quantified by scanning the $\mathrm{AgNO}_{3}$ positive area as described in Materials and methods. Data presented were average $\pm \mathrm{SD}$, $n=3 .{ }^{*} p<0.01$ and ${ }^{* *} p<0.001$ versus control group of $\mathrm{P} 1$, and ${ }^{\#} p<0.01$ and ${ }^{\# \#} p<0.001$ versus control group of P5.

propagation of cells and the contact inhibition of cells resulted in a slight increase of $\mathrm{p} 16^{\mathrm{INK} 4 \mathrm{a}}$ expression which agreed with what has been reported [18]. However, more than half of cells were shown to be BrdU-positive with minute amounts of cells being senescent. Thus, transferring cultures (CHA_P3/C and CHA_P5/C) were carried out, and CHA_P3/C demonstrated a further prolongation of cultural lifespan, while CHA_P5/C failed to prolong the lifespan, suggesting that pre-incubated with $\mathrm{CHA}$ for few passages (e.g., within 3 passages) may act to preserve the proliferative potentials of mADSCs.

In addition, upon the treatment of SHA and CHA, as well as the transfer culture, the osteogenic potential of mADSCs was preserved or even enhanced in comparison to control as shown in Fig. 4B, indicating that HA may act to keep mADSCs in an undifferentiated state prior to induction. Besides, the decrease of osteogenic potential 
with the increase of passage number in control group correlated well with the extent of cellular senescence, and the preservation of osteogenic potential by HA treatment may be a consequence of suppression of cellular senescence.

In this study, SHA was shown not only to promote the cell proliferation but preserve the osteogenic potential of mADSCs. Strikingly, pre-incubation with CHA for few passages would be more effective in prolonging the lifespan of mADSCs than SHA. Although the mechanism of HA in promoting proliferation and preserving the osteogenic potential of mADSCs need to be further investigated, the introduction of HA in a culture system may be useful for expanding adult stem cells in vitro without loss of their replicative and differentiation capacity.

\section{Acknowledgments}

This work was supported by grants of NSC94-2320-B006-046, NSC95-2320-B-002-052 NSC94-2214-E-002-011, and NSC95-2745-B-006-003-MY2 from National Science Council and grant of NHRI-EX91-8910EL from National Health Research Institutes of Taiwan.

\section{References}

[1] K.M. Safford, K.C. Hicok, S.D. Safford, Y.D. Halvorsen, W.O. Wilkison, J.M. Gimble, H.E. Rice, Neurogenic differentiation of murine and human adipose-derived stromal cells, Biochem. Biophys. Res. Commun. 294 (2002) 371-379.

[2] R. Ogawa, H. Mizuno, A. Watanabe, M. Migita, T. Shimada, H. Hyakusoku, Osteogenic and chondrogenic differentiation by adiposederived stem cells harvested from GFP transgenic mice, Biochem. Biophys. Res. Commun. 313 (2004) 871-877.

[3] R. Ogawa, H. Mizuno, A. Watanabe, M. Migita, H. Hyakusoku, T. Shimada, Adipogenic differentiation by adipose-derived stem cells harvested from GFP transgenic mice-including relationship of sex differences, Biochem. Biophys. Res. Commun. 319 (2004) 511-517.

[4] C.J. Sherr, R.A. DePinho, Cellular senescence: mitotic clock or culture shock? Cell 102 (2000) 407-410.

[5] W.E. Wright, J.W. Shay, Telomere dynamics in cancer progression and prevention: fundamental differences in human and mouse telomere biology, Nat. Med. 6 (2000) 849-851.

[6] S. Parrinello, E. Samper, A. Krtolica, J. Goldstein, S. Melov, J. Campisi, Oxygen sensitivity severely limits the replicative lifespan of murine fibroblasts, Nat. Cell Biol. 5 (2003) 741-747.

[7] T. Matsubara, S. Tsutsumi, H. Pan, H. Hiraoka, R. Oda, M. Nishimura, H. Kawaguchi, K. Nakamura, Y. Kato, A new technique to expand human mesenchymal stem cells using basement membrane extracellular matrix, Biochem. Biophys. Res. Commun. 313 (2004) 503-508.

[8] S.K. Nilsson, D.N. Haylock, H.M. Johnston, T. Occhiodoro, T.J. Brown, P.J. Simmons, Hyaluronan is synthesized by primitive hemopoietic cells, participates in their lodgment at the endosteum following transplantation, and is involved in the regulation of their proliferation and differentiation in vitro, Blood 101 (2003) 856-862.

[9] D. Peck, C.M. Isacke, CD44 phosphorylation regulates melanoma cell and fibroblast migration on, but not attachment to, a hyaluronan substratum, Curr. Biol. 6 (1996) 884-890.

[10] C.B. Knudson, Hyaluronan and CD44: strategic players for cellmatrix interactions during chondrogenesis and matrix assembly, Birth Defects Res. C Embryo Today 69 (2003) 174-196.

[11] M. Stojkovic, S. Kolle, S. Peinl, P. Stojkovic, V. Zakhartchenko, J.G. Thompson, H. Wenigerkind, H.D. Reichenbach, F. Sinowatz, E. Wolf, Effects of high concentrations of hyaluronan in culture medium on development and survival rates of fresh and frozen-thawed bovine embryos produced in vitro, Reproduction 124 (2002) 141-153.

[12] L. Huang, Y.Y. Cheng, P.L. Koo, K.M. Lee, L. Qin, J.C. Cheng, S.M. Kumta, The effect of hyaluronan on osteoblast proliferation and differentiation in rat calvarial-derived cell cultures, J. Biomed. Mater Res. A 66 (2003) 880-884.

[13] X. Zou, H. Li, L. Chen, A. Baatrup, C. Bunger, M. Lind, Stimulation of porcine bone marrow stromal cells by hyaluronan, dexamethasone and rhBMP-2, Biomaterials 25 (2004) 5375-5385.

[14] G.P. Dimri, X. Lee, G. Basile, M. Acosta, G. Scott, C. Roskelley, E.E. Medrano, M. Linskens, I. Rubelj, O. Pereira-Smith, et al., A biomarker that identifies senescent human cells in culture and in aging skin in vivo, Proc. Natl. Acad. Sci. USA 92 (1995) 93639367.

[15] P.A. Zuk, M. Zhu, H. Mizuno, J. Huang, J.W. Futrell, A.J. Katz, P. Benhaim, H.P. Lorenz, M.H. Hedrick, Multilineage cells from human adipose tissue: implications for cell-based therapies, Tissue Eng 7 (2001) 211-228.

[16] M. Pons, J.C. Cigudosa, S. Rodriguez-Perales, J.L. Bella, C. Gonzalez, C. Gamallo, M. Quintanilla, Chromosomal instability and phenotypic plasticity during the squamous-spindle carcinoma transition: association of a specific $\mathrm{T}(14 ; 15)$ with malignant progression, Oncogene 24 (2005) 7608-7618.

[17] C.S. Freitas, S.R. Dalmau, E. Abdelhay, Differential expression of notch signaling-related transcripts accompanies pro-thymocyte proliferation and phenotype transition induced by epidermal growth factor plus insulin in fetal thymus organ cultures, Mem. Inst. Oswaldo Cruz. 99 (2004) 381-388.

[18] R.J. Wieser, D. Faust, C. Dietrich, F. Oesch, p16INK4 mediates contact-inhibition of growth, Oncogene 18 (1999) 277-281.

[19] C.J. Collins, J.M. Sedivy, Involvement of the INK4a/Arf gene locus in senescence, Aging Cell 2 (2003) 145-150.

[20] S. Khaldoyanidi, J. Moll, S. Karakhanova, P. Herrlich, H. Ponta, Hyaluronate-enhanced hematopoiesis: two different receptors trigger the release of interleukin-1 beta and interleukin-6 from bone marrow macrophages, Blood 94 (1999) 940-949. 